Alma Mater Studiorum - Università di Bologna DEPARTMENT OF ECONOMICS

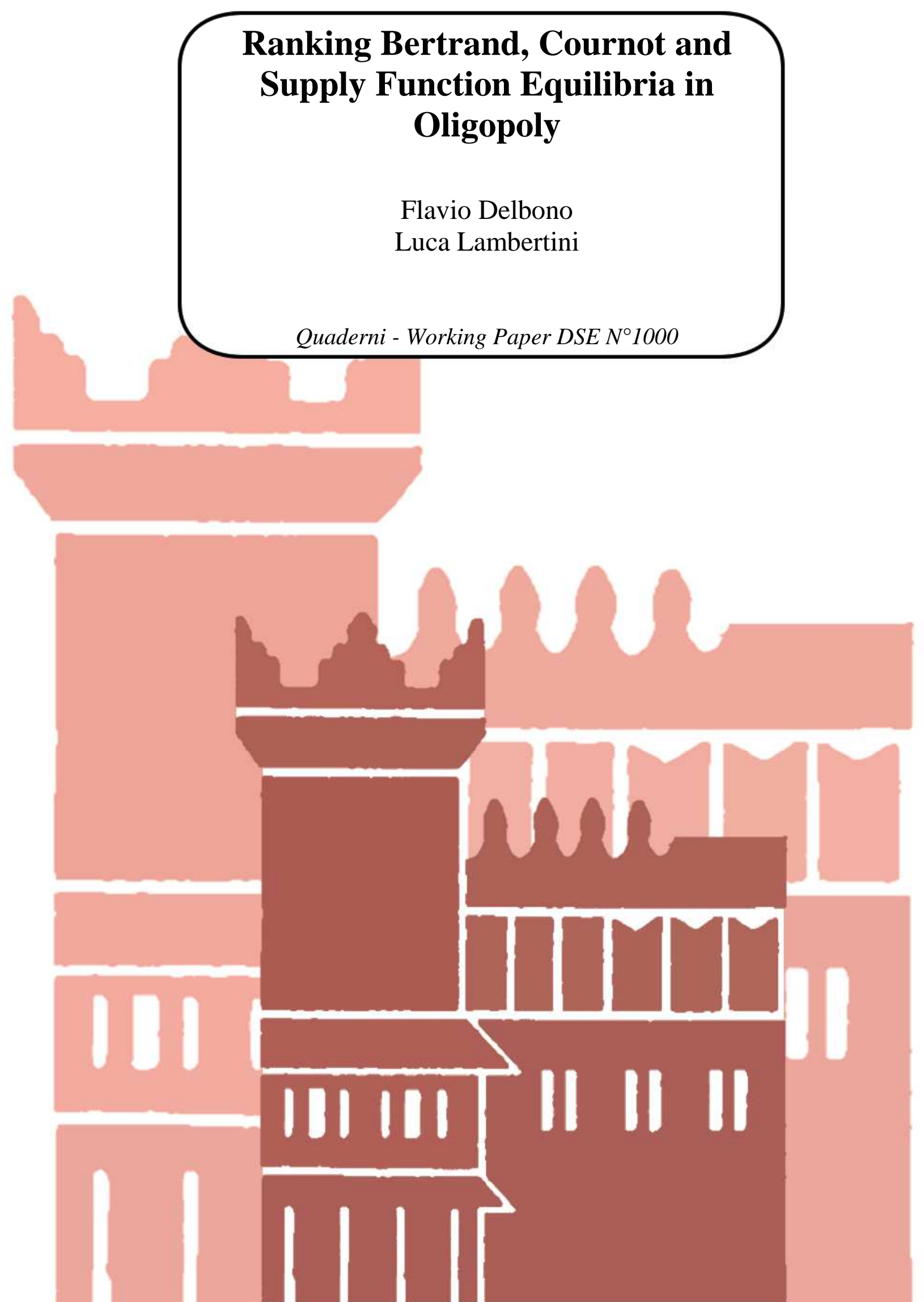




\title{
Ranking Bertrand, Cournot and Supply Function Equilibria in Oligopoly
}

\author{
Flavio Delbono ${ }^{\#}$ and Luca Lambertini ${ }^{\S}$ \\ \# Department of Economics, University of Bologna \\ Piazza Scaravilli 2, 40126 Bologna, Italy \\ flavio.delbono@unibo.it \\ $\S$ Department of Economics, University of Bologna \\ Strada Maggiore 45, 40125 Bologna, Italy \\ luca.lambertini@unibo.it
}

March 17, 2015 


\begin{abstract}
We show that the standard argument according to which supply function equilibria rank intermediate between Bertrand and Cournot equilibria may be reversed. We prove this result within a static oligopolistic game in which both supply function competition and Cournot competition yield a unique Nash equilibrium, whereas price setting yields a continuum of Nash equilibria. There are parameter regions in which Bertrand profits are higher than Cournot ones, with the latter being higher than in the supply function equilibrium. Such reversal of the typical ranking occurs when price-setting mimics collusion. We then show that the reversal in profits is responsible for a reversal in the welfare performance of the industry.
\end{abstract}

JEL Codes: D43, L13

Keywords: convex costs; supply function; price competition; quantity competition 


\section{Introduction}

There is little doubt about Cournot and Bertrand models being considered the most popular stylised representations of market games. However, there are markets characterised by sellers (and/or buyers) competing in supply (and/or demand) schedules: wholesale electricity, for instance, well fits such a setting in many countries. ${ }^{1}$ Despite the by now huge literature, it is still an open question "whether the price or quantity competition model is the better fit for different oligopolistic markets, and the supply function model appears to be an attractive model" (Vives, 2011, pp. 1919-20).

When modelling oligopolistic industries, an interesting question deals with ranking equilibria associated to different types of market competition. More precisely, one is likely interested in detecting and comparing predictions stemming - coeteris paribus - from equilibria in different strategies. If we confine the attention to Nash equilibria under the three aforementioned types of oligopolistic competition (quantities, prices, supply functions), since Klemperer and Meyer (1989) it has been claimed that the supply function equilibrium ranks intermediate between Bertrand and Cournot ones. Within static models of industries populated by identical firms producing homogeneous output, profits have been shown higher under quantity competition than under supply function competition, and higher in the latter setting than under Bertrand rules.

In this paper, we challenge such conclusion within a simple oligopoly game where Bertrand competition yields a continuum of Nash equilibria. We show that there exist parameter regions in which Bertrand profits are higher than Cournot ones, which in turn are higher than in the supply function equilibrium. the reversal in profit ranking drives a reversal in the standard welfare ranking. Intuitively, the reversals occur the higher the marginal cost and larger the departure from marginal cost pricing in the Bertrand game. In such a parameter constellation price-setting mimics collusive behaviour.

\footnotetext{
${ }^{1}$ See Klemperer and Meyer (1989) for other examples, and Grossman (1981). An excellent introduction to supply function equilibria is Vives (1999, ch. 7).
} 
The remainder of the paper is organised as follows. Section 2 summarises the related literature and locates our contribution in the research field. Section 3 presents the setup and the equilibria generated by the three different types of competition. Section 4 compares equilibrium profits and section 5 compares equilibrium levels of social welfare. Section 6 concludes.

\section{Related literature}

We may group the contributions closest to ours into three streams. The first one, starting from the mid 1980s, focusses on the comparison between the properties of Nash equilibria under price- and quantity-setting behaviour under different specification of technologies, demand and symmetry (or the lack thereof) across firms. A selection of the most quoted papers includes Singh and Vives (1984), Cheng (1985), Vives (1985), Okuguchi (1987), Qiu (1997), Häckner (2000), Zanchettin (2006). The focus of this discussion is about the relative performance of prices vs quantities in terms of profitability and social welfare, and the pivotal issue is the degree of symmetry across firms, especially in terms of productive technology, demand level and their interplay with product differentiation. The usual conclusion whereby Bertrand is less profitable and more efficient than Cournot can flip over in presence of a sufficiently high degree of cost and demand asymmetry (see Zanchettin, 2006). ${ }^{2}$ The entire discussion taking place in this subset of the literature considers models delivering unique equilibria in the relevant strategic variable.

The second stream of literature has been pioneered by Dastidar (1995) who has proved the existence of a continuum of pure-strategy equilibrium prices in an homogeneous oligopoly. The properties of Bertrand-Nash equilibria have been investigated also by Dastidar $(1997,2011)$ under different specifications of the cost functions, under the assumption of product homogeneity. The interesting paper by Saporiti and Coloma (2010) presents new

\footnotetext{
${ }^{2}$ An analogous reversal obtains in dynamic games, for instance when resource extraction enters the picture, as in Colombo and Labrecciosa (2015).
} 
results and an extremely helpful taxonomy of the most recent contributions on price competition. The focus of this portion of the literature is about the uniqueness of pure (or mixed) strategy equilibrium price depending on the specification of the cost function, also including fixed components.

Finally, there is a smaller group of papers concerned with competition in supply functions. This literature was initiated by Grossman (1981) and especially Klemperer and Meyer (1989), who consider an oligopolistic game with demand uncertainty. More recent papers include Delgado and Moreno (2004) and Ciarreta and Gutierrez-Hita (2006), to which we shall refer again later.

Our contribution crosses the aforementioned streams as we compare Bertrand, Cournot and supply functions equilibria in an industry where price-setting yields a continuum of Bertrand equilibria in pure strategies, the good is homogeneous, all firms are endowed with the same technology displaying increasing variable costs and no fixed ones.

\section{Setup and the three games}

Here we describe an industry by means of assumptions that make tractable a model otherwise very complex, especially as for then case in which strategies are functions (the supply function case). Moreover, our functional specification of demand and technology will allow us a complete comparison of the three types of market games equilibria.

Consider a market supplied by a set $\mathcal{N}=1,2,3, \ldots, n$ of identical firms producing a homogeneous good whose direct demand function is $Q=\max \{0,1-p\}$, where $Q=\sum_{i=1}^{n} q_{i}$ is aggregate output, $q_{i}$ is firm $i$ 's output and $p$ is price. Production takes place at decreasing returns to scale, and technology, shared by all firms, is summarised by the strictly convex cost function $C_{i}=c q_{i}^{2} / 2$. Accordingly, the profit function of firm $i$ is

$$
\pi_{i}=\left(p-\frac{c q_{i}}{2}\right) q_{i}=\left(1-q_{i}-Q_{-i}-\frac{c q_{i}}{2}\right) q_{i}
$$

where $Q_{-i}=\Sigma_{j \neq i} q_{j}$. 
Firms play simultaneously a non-cooperative one-shot game under complete, symmetric and imperfect information. The solution concept is the Nash equilibrium.

- Under Cournot competition, the relevant first order condition (FOC) for firm $i$ is the following:

$$
\frac{\partial \pi_{i}}{\partial q_{i}}=1-2 q_{i}-Q_{-i}-c q_{i}=0
$$

and the symmetry condition $\left(q_{j}=q_{i}=q\right.$ for all $i$ and $\left.j\right)$ yields the unique Cournot-Nash individual equilibrium output and price

$$
q^{C N}=\frac{1}{n+1+c} ; p^{C N}=\frac{1+c}{n+1+c}
$$

The resulting equilibrium profits are

$$
\pi^{C N}=\frac{2+c}{2(n+1+c)^{2}}
$$

- In modelling the price-setting game, we follow Dastidar (1995), where it is shown that, if costs are strictly convex in output levels and demand is decreasing in price, Bertrand competition yields a continuum of Nash equilibria when firms are identical. More precisely, the Nash equilibrium in pure strategies involves all firms setting the same price $p^{*} \in\left[p^{a v c}, p^{u}\right]$. At the lower bound $p^{a v c}$, equilibrium price equals average variable costs, so that firms would be indifferent between producing or not. At the upper bound $p^{u}$, the equilibrium price is such that firms would be indifferent between playing $p^{u}$ or marginally undercutting it in order to serve the entire market demand.

Without delving further into the details of the derivation of the continuum of price equilibria (see Dastidar, 1995, pp. 27-28; and Gori et al. 2014, pp. 373-75), the spectrum of equilibrium prices is identified by

$$
p^{B N}=\frac{c}{c+2(n-\alpha)}
$$


where $\alpha$ is a non-negative parameter whose range, to be specified below, determines the continuum of equilibrium prices. The associated individual output is

$$
q^{B N}=\frac{2(n-\alpha)}{n[c+2(n-\alpha)]}
$$

and profits are

$$
\pi^{B N}=\frac{2 \alpha c(n-\alpha)}{n^{2}[c+2(n-\alpha)]^{2}} .
$$

As far as parameter $\alpha$ is concerned, it is worth noting that:

1. in $\alpha=0$, the equilibrium price equals average variable cost;

2. at $\alpha=n / 2$, marginal cost pricing obtains;

3. if $\alpha=n^{2} /(1+n), p^{B N}$ reaches the highest level above which undercutting takes place.

Consequently, the admissible range is $\alpha \in\left[0, n^{2} /(1+n)\right]$. For future reference, we define $\alpha_{\text {sup }}:=n^{2} /(1+n)$.

The following result will become useful in the remainder:

Lemma $1 p^{B N}>p^{C N}$ for all $\alpha>\alpha_{B C}^{p} \equiv n(2+c) /[2(1+c)]$, with $\alpha_{B C}^{p} \in$ $\left(n / 2, n^{2} /(n+1)\right]$ for all $c \geq 2 /(n-1)$.

Proof. The difference between the two equilibrium prices is

$$
p^{B N}-p^{C N}=\frac{2 \alpha(1+c)-n(2+c)}{(n+1+c)[2(n-\alpha)+c]}
$$

where the denominator is positive since $\alpha \leq \alpha_{\text {sup }}<n$. Hence, the sign of (8) is the sign of the expression appearing at the numerator, which is positive for all $\alpha>n(2+c) /[2(1+c)] \equiv \alpha_{B C}^{p}$. It is then easily ascertained that $\alpha_{B C}^{p} \in\left(n / 2, n^{2} /(n+1)\right]$ for all $c \geq 2 /(n-1)$.

The interpretation of the above Lemma is that Bertrand equilibrium price exceeds the Cournot one in the admissible range of $\alpha$ when the cost function is sufficiently steep and $\alpha$ is such that the Bertrand-Nash price departs enough from marginal cost. 
- In modelling supply function competition, we adopt the same approach used by Ciarreta and Gutierrez-Hita (2006), which offers a simple tool for solving the supply function game originally formulated by Klemperer and Meyer (1989). Given the linear-quadratic form of the profit function (1), one may formulate the conjecture that supply functions are linear in price. Therefore, we define the supply function of firm $i$ as $s_{i}=\beta_{i} p, \beta_{i}>0$. The market clearing price solves

$$
1-p=\sum_{i=1}^{n} s_{i}
$$

from which we have

$$
p=\frac{1}{1+\sum_{i=1}^{n} \beta_{i}}
$$

Hence, we may rewrite the individual profit function as follows:

$$
\pi_{i}=\frac{\beta_{i}}{\left(1+\sum_{i=1}^{n} \beta_{i}\right)^{2}}\left(1-\frac{c \beta_{i}}{2}\right)
$$

This amounts to saying that, if firms compete in supply functions, their set of strategic variables is the vector of $\beta_{i}$ 's, one for each firm. The FOC is:

$$
\frac{\partial \pi_{i}}{\partial \beta_{i}}=\frac{1+\sum_{j \neq i} \beta_{j}-\beta_{i}\left[1+c\left(1+\sum_{j \neq i} \beta_{j}\right)\right]}{\left(1+\sum_{i=1}^{n} \beta_{i}\right)^{3}}=0
$$

Imposing symmetry across firms, as the second order condition is met, the unique equilibrium strategy solving the above equation is

$$
\beta^{S F N}=\frac{n-2-c+\sqrt{(n-2)^{2}+c(2 n+c)}}{2 c(n-1)}
$$

which is clearly positive for all admissible values of $c$ and $n$. The associated equilibrium price, individual output and profits are

$$
p^{S F N}=\frac{2 c(n-1)}{c(n-2)+n\left(n-2+\sqrt{(n-2)^{2}+c(2 n+c)}\right)}
$$




$$
\begin{gathered}
q^{S F N}=\frac{n+2+c-\sqrt{(n-2)^{2}+c(2 n+c)}}{2(2 n+c)} \\
\pi^{S F N}=\frac{2 n+(n+c)\left(\sqrt{(n-2)^{2}+c(2 n+c)}-n-c\right)}{4(2 n+c)}
\end{gathered}
$$

with $q^{S F N}$ and $\pi^{S F N}$ being strictly positive over the entire parameter range.

\section{Ranking equilibrium profits}

In Klemperer and Meyer (1989, pp. 1258-59), it is shown that the equilibrium profits generated by competition in supply functions are intermediate between those generated by Cournot and Bertrand behaviour, when the latter is restricted to marginal cost pricing. ${ }^{3}$

Proposition 2 (Klemperer and Meyer, 1984, p. 1259) $\pi^{C N}>\pi^{S F N}$ for all $n \geq 2$.

Without repeating the full proof, it suffices to note that, in our model, the sign of $\pi^{C N}-\pi^{S F N}$ is the sign of

$$
(n-1)^{2}(2 n+c)\left[2 c^{2}+2 n(n+2)+c(4 n+3)\right]
$$

as can be easily ascertained using (4) and (16); the sign of the above expression is clearly positive.

Under marginal cost pricing, it would also be true that $\pi^{C N}>\pi^{S F N}>$ $\pi^{B N}$ for all $n \geq 2$, as in Klemperer and Meyer (1989). However, here we are dealing with a continuum of price equilibria ranging well above marginal

\footnotetext{
${ }^{3} \mathrm{~A}$ similar conclusion is reached by Delgado and Moreno (2014, Theorem 2.2), in a model without uncertainty where supply functions are bound to be non-decreasing, demand is strictly decreasing and convex, costs are non-drecreasing, convex and identical across firms.
} 
cost pricing. Since Betrand-Nash equilibrium profits $\pi^{B N}$ are a function of $\alpha$, our strategy consists in searching for admissible intervals of $\alpha$ in which $\pi^{B N}$ overcomes $\pi^{S F N}$ and even $\pi^{C N}$.

We start by comparing $\pi^{B N}$ and $\pi^{C N}$. The expression

$\pi^{B N}-\pi^{C N}=-\frac{[(2+c) n-2(1+c) \alpha]\left[n(2 n+c)^{2}-2(c(1+c)+2 n(c+n)) \alpha\right]}{2 n^{2}(n+1+c)^{2}[c+2(n-\alpha)]^{2}}$

is positive for all $\alpha \in\left(\alpha_{B C 1}^{\pi}, \alpha_{B C 2}^{\pi}\right)$, with

$$
\alpha_{B C 1}^{\pi}=\frac{(2+c) n}{2(1+c)} ; \alpha_{B C 2}^{\pi}=\frac{n(2 n+c)^{2}}{2[c(1+c)+2 n(c+n)]}
$$

Now note that

$$
\begin{gathered}
\lim _{c \rightarrow 0} \alpha_{B C 1}^{\pi}=\lim _{c \rightarrow 0} \alpha_{B C 2}^{\pi}=n \\
\alpha_{\text {sup }}>\alpha_{B C 1}^{\pi} \forall c>c_{B C 1}=\frac{2}{n-1}
\end{gathered}
$$

and

$$
\alpha_{\text {sup }}>\alpha_{B C 2}^{\pi} \forall c>c_{B C 2}=\frac{n(1+\sqrt{4 n-3})}{n-1}>c_{B C 1} .
$$

Since $\pi^{C N}>\pi^{S F N}$ from Proposition 1, this immediately implies:

Proposition $3 \pi^{B N}>\pi^{C N}>\pi^{S F N}$ for all $\alpha \in\left(\alpha_{B C 1}^{\pi}, \min \left\{\alpha_{B C 2}^{\pi}, \alpha_{\text {sup }}\right\}\right)$.

The relevant region is drawn in Figure 1, in the space $(c, \alpha)$. 
Figure $1 \pi^{B N}>\pi^{C N}>\pi^{S F N}$ in the space $(c, \alpha)$

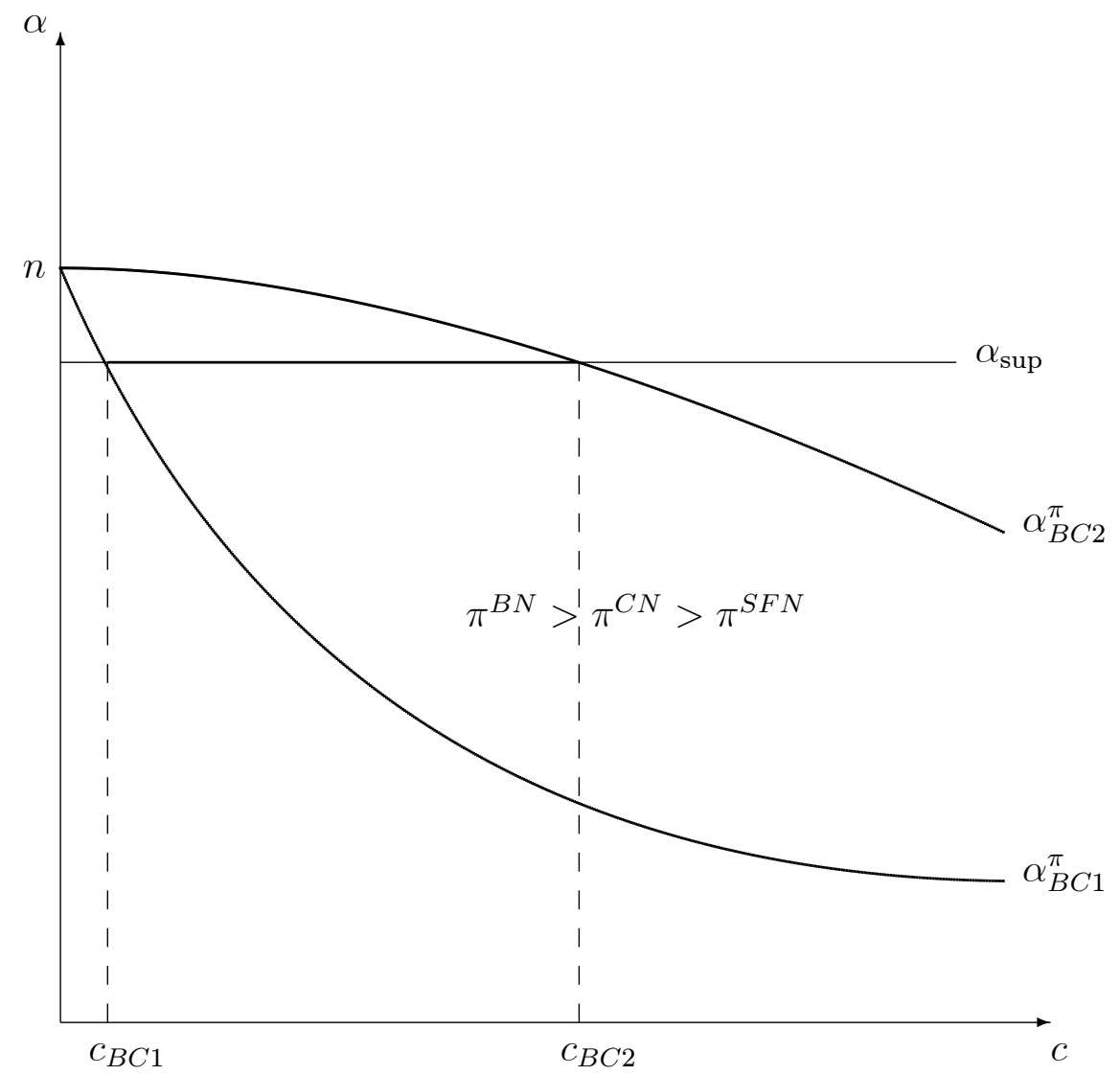

We now show that the region in which $\pi^{B N}>\pi^{C N}>\pi^{S F N}$ is a proper subset of the parameter range wherein $\pi^{B N}>\pi^{S F N}$. To do so, we have to compare $\pi^{B N}$ and $\pi^{S F N}$. Tedious algebra is needed to verify that

$$
\pi^{B N}>\pi^{S F N} \forall \alpha \in\left(\alpha_{B S F 1}^{\pi}, \min \left\{\alpha_{B S F 2}^{\pi}, \alpha_{\text {sup }}\right\}\right)
$$

with

$$
\alpha_{B S F 1}^{\pi}=\frac{n(2 n+c)\left[c^{2} n+n^{2}(n-2-x)-c(2-y-n(2 n-x))\right]}{2\left[c^{2}\left(n^{2}-2\right)+n^{3}(n-2-x)+n c(n(2 n-x)-4)\right]}
$$




$$
\alpha_{B S F 2}^{\pi}=\frac{n(2 n+c)\left[c^{2} n+n^{2}(n-2-x)-c(2+y-n(2 n-x))\right]}{2\left[c^{2}\left(n^{2}-2\right)+n^{3}(n-2-x)+n c(n(2 n-x)-4)\right]}
$$

where

$$
\begin{gathered}
x:=\sqrt{c(c+2 n)+(n-2)^{2}} \\
y:=\sqrt{2[(n+c)(n+c-x)-2(n-1)]}
\end{gathered}
$$

in such a way that, indeed, $\alpha_{B S F 2}^{\pi}>\alpha_{B S F 1}^{\pi}$ and $\lim _{c \rightarrow 0} \alpha_{B S F 1}^{\pi}=\lim _{c \rightarrow 0} \alpha_{B S F 2}^{\pi}=$ $n$. The region where $\pi^{B N}>\pi^{S F N}$, identified in (23), is drawn in Figure 2.

Figure $2 \pi^{B N}>\pi^{S F N}$ in the space $(c, \alpha)$

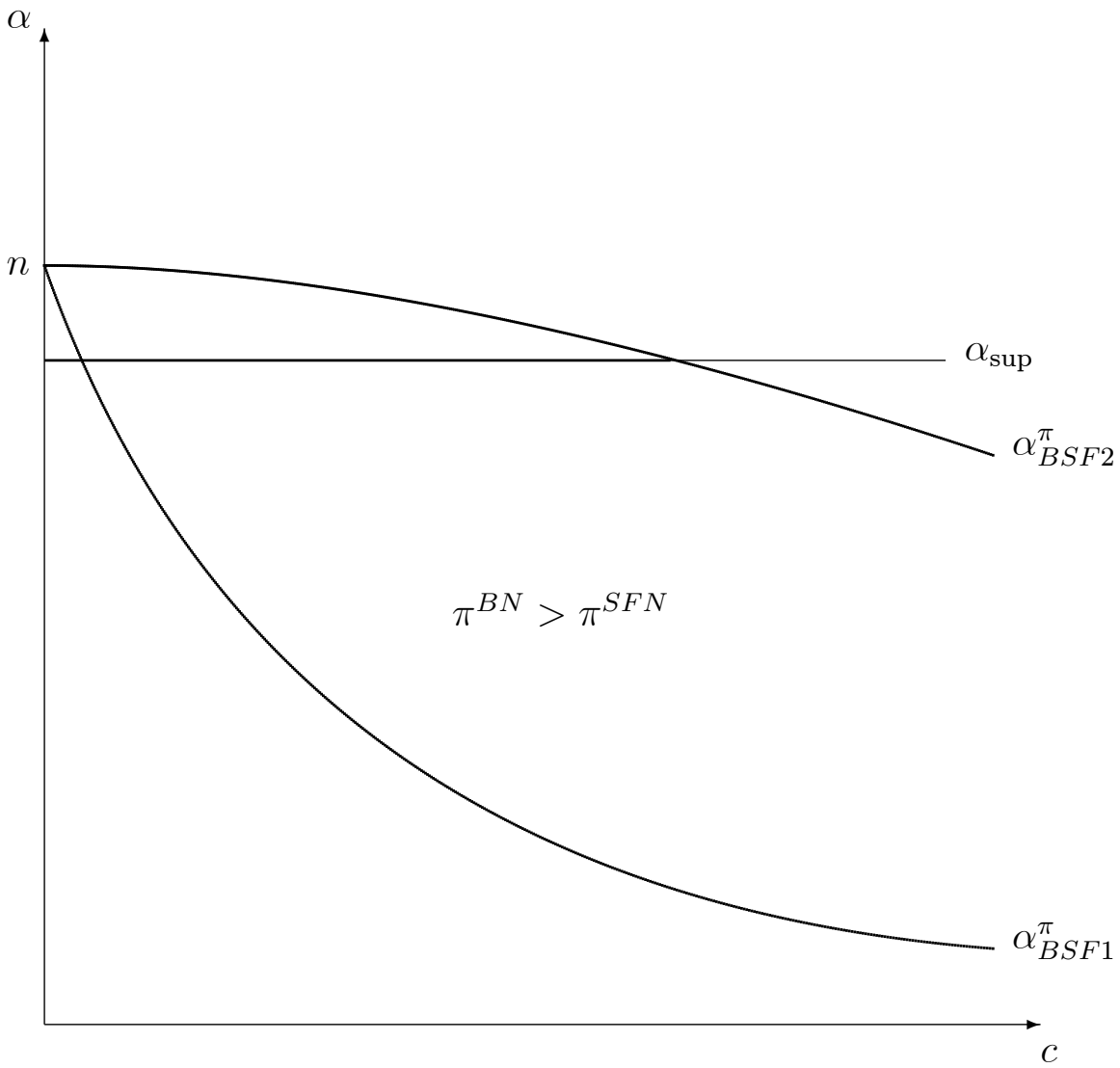


We may now order the critical thresholds of $\alpha$ appearing in Figures 1-2. The difference between $\alpha_{B S F 2}^{\pi}$ and $\alpha_{B C 2}^{\pi}$ is:

$\alpha_{B S F 2}^{\pi}-\alpha_{B C 2}^{\pi}=\frac{c n(c+2 n) y}{c n\left(n x+4-2 n^{2}\right)-n^{3}(n-2-x)-c^{2}\left(n^{2}-2\right)}>0 \forall c>0, n \geq 2$.

By the same token, one can verify that $\alpha_{B C 1}^{\pi}>\alpha_{B S F 1}^{\pi}$ in the same space $\{c, n\}$. This delivers Figure 3 , where the set of curves $\left\{\alpha_{B S F 1}^{\pi}, \alpha_{B S F 2}^{\pi}, \alpha_{B C 1}^{\pi}, \alpha_{B C 2}^{\pi}\right\}$ are drawn.

Figure 3 Ranking equilibrium profits in the space $(c, \alpha)$

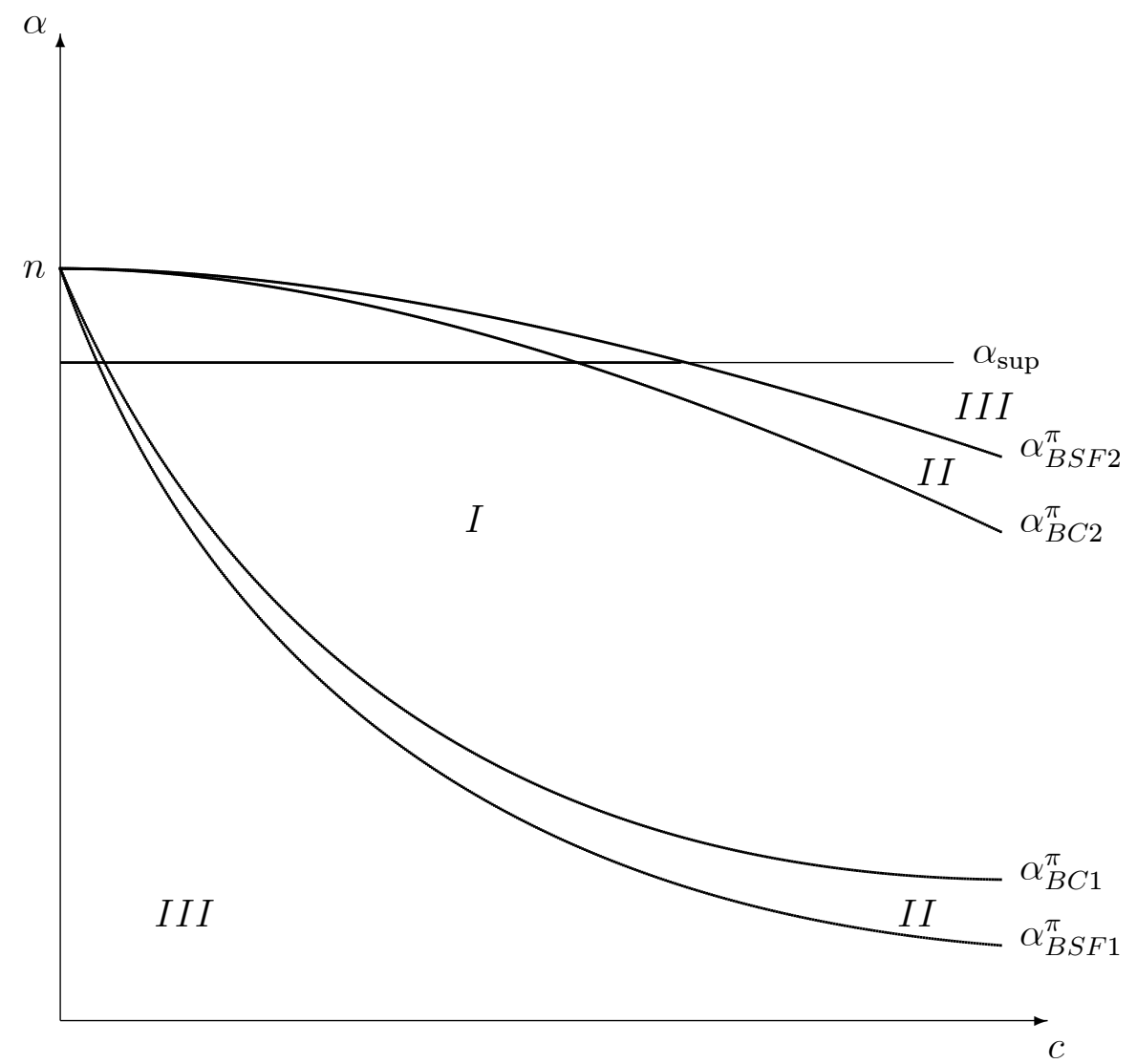


In Figure 3, we identify three regions:

- Region $I$, where $\pi^{B N}>\pi^{C N}>\pi^{S F N}$

- Region $I I$, where $\pi^{C N}>\pi^{B N}>\pi^{S F N}$

- Region $I I I$, where $\pi^{C N}>\pi^{S F N}>\pi^{B N}$

Region III hosts the familiar ranking seeing the supply function equilibrium as intermediate between Bertrand and Cournot equilibria. In Region $I I$, Bertrand equilibrium profits overcome those generated by supply function competition. In region $I$, we have a full reversal of the traditional profit ranking.

The intuition behind the ranking in region $I$ can be explained as follows. This region is featured by (comparatively) high values of both $\alpha$ and c. Recalling Lemma 1, one may explain the chain of inequalities emerging in region $I$ on the basis of the inequality between $p^{B N}$ and $p^{C N}$. When $\alpha$ and $c$ are high enough, the Bertrand-Nash price ranks first, price-setting firms implementing a quasi-collusive outcome.

In region $I$, Bertrand behaviour outperforms both Cournot and supply function competition from the firms' standpoint. One may then conjecture that, for any $c>c_{B C 1}$, if $\alpha$ is larger than $\alpha_{B C 1}^{\pi}$, welfare levels rank opposite to profits. This is indeed what we are about to show in the next section.

\section{$5 \quad$ Ranking equilibrium welfare levels}

Define social welfare as $S W^{K N}=n \pi^{K N}+C S^{K N}$, where $K=B, C, S F$ and $C S^{K N}=\left(Q^{K N}\right)^{2} / 2$ is consumer surplus, defined in terms of industry output $Q^{K N}=n q^{K N}$. Since the welfare level is proportional to the industry output, for the sake of simplicity we may restrict our attention to industry outputs across equilibria. To begin with, we compare $Q^{S F N}$ against $Q^{C N}$ :

$$
\operatorname{sign}\left\{Q^{S F N}-Q^{C N}\right\}=\operatorname{sign}\{(n-1)(2 n+c)\}
$$


which is positive everywhere. Then,

$$
Q^{B N} \gtreqless Q^{C N} \text { for all } \alpha \lesseqgtr \frac{(2+c) n}{2(1+c)}=\alpha_{B C 1}^{\pi}
$$

Taken together, (29-30) imply:

Proposition 4 Take $c>c_{B C 1}$. As soon as firms set $\alpha>\alpha_{B C 1}^{\pi}$, we have $Q^{S F N}>Q^{C N}>Q^{B N}$. As a result, $S W^{S F N}>S W^{C N}>S W^{B N}$.

The above Proposition has a natural explanation, in that when Bertrand profits rank first, this happens through an output restriction and a price increase, which of course is detrimental to welfare.

\section{Concluding remarks}

In this paper, we have presented a simple linear-quadratic model of homogeneous oligopoly allowing a fully-fledged comparative analysis of different market games. We have shown that the standard ranking among, price, quantity and supply function equilibria may be reversed for profits as well as social welfare levels. The reason why equilibrium profits (welfare) may be higher (lower) under price competition than under quantity and supply function competition lies in firms being able to price well above marginal cost in the Bertrand game. Such an ability stems from the convexity of the cost function and the resulting continuum of pure-strategy equilibrium prices. This reversal of the standard profit and welfare rankings occur when price competition mimics collusive behaviour. Hence, the convexity of the cost function may prevent ranking the supply function equilibrium as intermediate between price and quantity equilibria. 


\section{References}

[1] Cheng, L. (1985), "Comparing Bertrand and Cournot Equilibria: A Geometric Approach", RAND Journal of Economics, 16, 146-52.

[2] Ciarreta, A. and C. Gutierrez-Hita (2006), "Supply Function vs Quantity Competition in Supergames", International Journal of Industrial Organization, 24, 773-83.

[3] Colombo, L. and P. Labrecciosa (2015), "On the Markovian Efficiency of Bertrand and Cournot Equilibria", Journal of Economic Theory, 155, $332-58$.

[4] Dastidar, K.G. (1995), "On the Existence of Pure Strategy Bertrand Equilibrium", Economic Theory, 5, 9-32.

[5] Dastidar, K.G. (1997), "Comparing Cournot and Bertrand in a Homogeneous Product Market", Journal of Economic Theory, 75, 205-12.

[6] Dastidar, K.G. (2011), "Existence of Bertrand Equilibrium Revisited", International Journal of Economic Theory, 7, 331-50.

[7] Delgado, J. and D. Moreno (2004), "Coalition-Proof Supply Function Equilibria in Oligopoly", Journal of Economic Theory, 114, 231-54.

[8] Grossman, S. (1981), "Nash Equilibrium and the Industrial Organization of Markets with Large Fixed Costs", Econometrica, 49, 1149-72.

[9] Häckner, J. (2000), "A Note on Price and Quantity Competition in Differentiated Oligopolies", Journal of Economic Theory, 93, 233-39.

[10] Klemperer, P.D. and M.A. Meyer (1989), "Supply Function Equilibria in Oligopoly under Uncertainty", Econometrica, 57, 1243-77.

[11] Gori, G., L. Lambertini and A. Tampieri (2014), "Trade Costs, FDI Incentives, and the Intensity of Price Competition", International Journal of Economic Theory, 10, 371-85. 
[12] Menezes, F.M. and J. Quiggin (2012), "More Competitors or More Competition? Market Concentration and the Intensity of Competition", Economics Letters, 117, 712-14.

[13] Okuguchi, K. (1987), "Equilibrium Prices in the Bertrand and Cournot Oligopolies", Journal of Economic Theory, 42, 128-39.

[14] Qiu, L. (1997), "On the Dynamic Efficiency of Bertrand and Cournot Equilibria", Journal of Economic Theory, 75, 213-29.

[15] Saporiti, A. and G. Coloma (2010), "Betrand Competition in Markets with Fixed Costs", B.E. Journal of Theoretical Economics (Contributions), 10, article 27.

[16] Singh, N. and X. Vives (1984), "Price and Quantity Competition in a Differentiated Duopoly", RAND Journal of Economics, 15, 546-54.

[17] Vives, X. (1985), "On the Efficiency of Bertrand and Cournot Equilibria with Product Differentiation", Journal of Economic Theory, 36, 166-75.

[18] Vives, X. (1999), Oligopoly Pricing: Old Ideas and New Tools, Cambridge, MA, MIT Press.

[19] Vives, X. (2011), "Strategic Supply Function Competition with Private Information", Econometrica, 79, 1919-66.

[20] Zanchettin, P. (2006), "Differentiated Duopoly with Asymmetric Costs", Journal of Economics and Management Strategy, 15, 999-1015. 


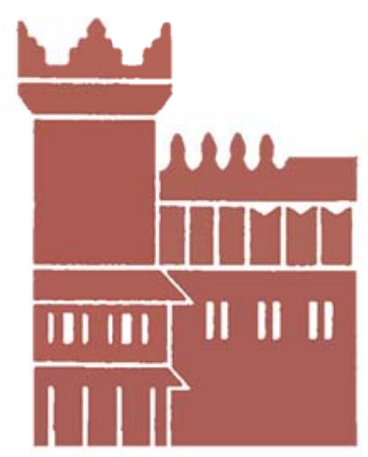

Alma Mater Studiorum - Università di Bologna DEPARTMENT OF ECONOMICS

Strada Maggiore 45

40125 Bologna - Italy

Tel. +39051 2092604

Fax +390512092664

http://www.dse.unibo.it 